\title{
Studies on Physiological and Hemato-Biochemical Changes during Xylazine-Zolazepam + Tiletamine and Dexmedetomidine - Zolazepam + Tiletamine Anesthesia in Sloth Bears
}

\author{
M.M. Anitha, L. Ranganath, H.S. Shwetha*, Arun Sha, B.P. Shankar, \\ V. Mahesh and R.B. Srinivas
}

Institute of Wildlife Veterinary Research, Doddaluvara, Kodagu Veterinary College, Hebbal, Bengaluru, Karnataka Veterinary, Animal and Fisheries, Sciences University, Bidar, India

*Corresponding author

\section{A B S T R A C T}

\begin{abstract}
Keywords
Sloth bears,

Physiological, Hemato-

Biochemical changes,

XZT, DexMZT,

anesthesia

Article Info

Accepted:

24 September 2018

Available Online:

10 December 2018

\section{Introduction}

Sloth bears are classified as Vulnerable in IUCN red list of Threatened species in 2008. Although bears are not difficult to anesthetize, it is better to be cautious as they are monogastrics and prone to vomiting on induction or regurgitation during anesthesia (Caulkett and Cattet, 2002) which could be dangerous.
\end{abstract}

The physiological and hemato-biochemical changes were recorded at 15, 30, and 60 minutes after immobilization with XZT (group A) and DexMZT (group B) in six sloth bears of each group. Significant physiological difference in heart rate between the groups at 30 minutes was observed which could be due to $\alpha_{2}$ agonist mediated biphasic cardiovascular response causing bradycardia. Significant decrease in hemoglobin concentration at 30 minutes in group B could be attributed to $\alpha_{2}$ agonist influencing shifting of fluids from extracellular to intracellular compartments. Significant difference in neutrophil count can be attributed to DexM showing very less stress response. No significant changes were observed in biochemical values.
In the present study, the complications of anesthesia including the physiological, hemato-biochemical assessments and side effects were considered for a safer immobilization in captive sloth bears.

\section{Materials and Methods}

Twelve sloth bears of either gender were randomly selected for immobilization in Wildlife SOS, Bannerghatta Bear Rescue Centre at Bengaluru.

The bears were divided into two groups $\mathrm{A}$ and $B$ of six each. Group A and B were immobilized with XZT and DexMZT respectively. Physiological parameters (Rectal 
temperature $\quad{ }^{0} \mathrm{~F} ; \quad$ Respiratory rate breath/minute; Heart rate- beats/ minute) were recorded using OT patient Monitor as suggested by Ozeki et al., (2014) at 15, 30, 60 minutes after immobilization. 0 minute/Normal values were referred as per book standards (Fowler, 1978).

Hematological and Biochemical parameters were analyzed at 15, 30, 60 minutes using Mindray BC-2800 Vet auto analyzer and Thermo scientific Konelab-20 fully automated biochemical analyzer respectively. Ominute/Normal values were referred as per book standards (Miller and Fowler, 2003).

The Mean and Standard error of the data were analyzed by t-test using computer based statistical programme.

\section{Results and Discussion}

Mean values of rectal temperature recorded at $15,30,60$ minutes were $99.48 \pm 0.19,100.1 \pm$ $0.21,99.82 \pm 0.14{ }^{0} \mathrm{~F}$ respectively. Respiratory rate were $20.50 \pm 2.34,16.83 \pm 0.75$, $23.50 \pm 1.69$ breaths / minute respectively and Heart rate were $66.17 \pm 6.26,76.50 \pm 5.92$ and $77.83 \pm 4.11$ beats / minute respectively.

Although all the values were within the normal range a non-significant rise in rectal temperature could be due to vasoconstriction of peripheral vessels caused by $\alpha_{2}$ agonist, it did not lead to hyperthermia as stated by Cattet et al., (2003a) and Cattet et al., (2003b).

Since $\alpha_{2}$ agonist induce hypoxemia regular monitoring is needed and if necessary oxygen supplementation can be provided as suggested by (Cattet et al., 2003c., Rai, 2009). The respiratory values in group B were within the normal range similar to reports of by Neto, (2009); Teisberg et al., (2014) and Jin et al., (2016) and DexM had organ protective effect against ischemic and hypoxic injury as stated by Alfonso and Reis, (2012). A significant difference in heart rate $(\mathrm{P}<0.05)$ was found at 30 minutes although the values were within normal range, similar reports were indicated by Ansah, (2004) Neto, (2009) and Teisberg et al., (2014) where mild bradycardia induced by DexM can be tolerated by healthy animals with no cardiovascular disease.

Alfonso and Reis, (2012) stated that DexM provides Reno protection, Neuro protection and Cardio protection.

The Mean \pm SE of TEC in group A increased slightly at 30 minutes which could be attributed to the effect of $\alpha_{2}$ agonist causing splenic contractions as reported by Ganong, (2002).

TLC count in group B consisting two cubs and a subadult bears were higher compared to group A which could be due maturation of immune system as stated by Arun et al., (2008).

At 30 minutes after immobilization there was a significant $(\mathrm{P}<0.05)$ decrease in hemoglobin concentration and $(\mathrm{P}<0.01)$ decrease in $\mathrm{PCV}$ values in group $B$. This could be due to $\alpha_{2}$ agonists influencing shifting of fluids from extracellular to intracellular compartments to maintain cardiac output and the difference is more in group B as DexM has a property to preserve blood flow to vital organs as reported by Rafee et al., (2015).

Differential leucocyte counts are varied in both groups, this could be due to the different age groups and sex as stated by (Arun et al., 2008; Graesili et.al. 2014). A significant difference in neutrophil count at 30 minutes between the groups may be attributed to very less stress response in group B due to faster and smoother induction of DexM causing less physical exertion which is similar to reports of Rafee et al., (2015) (Table 1-4). 
Table.1 Mean \pm SE values of Hematological parameters of sloth bears in Group A and B

\begin{tabular}{|c|c|c|c|c|c|c|c|}
\hline \multirow[t]{2}{*}{ Parameters } & \multirow{2}{*}{$\begin{array}{c}\text { (Miller and } \\
\text { Fowler, 2003) } \\
\text { 0 min/Normal } \\
\text { range }\end{array}$} & \multicolumn{3}{|c|}{ Group A } & \multicolumn{3}{|c|}{ Group B } \\
\hline & & $\begin{array}{c}15 \\
\text { minutes }\end{array}$ & $\begin{array}{c}30 \\
\text { minutes }\end{array}$ & $\begin{array}{c}60 \\
\text { minutes }\end{array}$ & $\begin{array}{c}15 \\
\text { minutes }\end{array}$ & $\begin{array}{c}30 \\
\text { minutes }\end{array}$ & $\begin{array}{c}60 \\
\text { minutes }\end{array}$ \\
\hline $\begin{array}{l}\text { TEC }\left(\times 10^{6} /\right. \\
\text { ul) }\end{array}$ & $5.58-14.70$ & $\begin{array}{c}5.56 \pm \\
0.23^{\mathrm{a}}\end{array}$ & $\begin{array}{c}5.92 \pm \\
0.17^{\mathrm{a}}\end{array}$ & $\begin{array}{l}6.11 \pm \\
0.07^{\mathrm{a}}\end{array}$ & $\begin{array}{c}5.82 \pm \\
0.18^{\mathrm{a}}\end{array}$ & $\begin{array}{c}5.97 \pm \\
0.17^{\mathrm{a}}\end{array}$ & $\begin{array}{l}6.11 \pm \\
0.08^{\mathrm{a}}\end{array}$ \\
\hline $\begin{array}{l}\text { TLC }\left(\times 10^{3} /\right. \\
\mu l)\end{array}$ & $5.90-24.0$ & $\begin{array}{c}12.92 \pm \\
0.91^{\mathrm{a}}\end{array}$ & $\begin{array}{c}12.28 \pm \\
1.10^{\mathrm{a}}\end{array}$ & $\begin{array}{c}13.28 \pm \\
0.85^{\mathrm{a}}\end{array}$ & $\begin{array}{c}14.63 \pm \\
0.76^{\mathrm{a}}\end{array}$ & $\begin{array}{c}13.45 \pm \\
0.97^{\mathrm{a}}\end{array}$ & $\begin{array}{c}14.60 \pm \\
0.80^{\mathrm{a}}\end{array}$ \\
\hline $\begin{array}{l}\text { Hemoglobin } \\
\text { (g/ dl) }\end{array}$ & $8.5-20.4$ & $\begin{array}{c}14.50 \pm \\
0.35^{\mathrm{a}}\end{array}$ & $\begin{array}{c}13.92 \pm \\
0.27^{\mathrm{a}}\end{array}$ & $\begin{array}{c}15.27 \pm \\
0.18^{\mathrm{a}}\end{array}$ & $\begin{array}{c}14.33 \pm \\
0.51^{\mathrm{a}}\end{array}$ & $\begin{array}{c}13.88 \pm \\
0.59^{\mathrm{a}}\end{array}$ & $\begin{array}{c}15.10 \pm \\
0.21^{\mathrm{a}}\end{array}$ \\
\hline PCV (\%) & $35.0-54.0$ & $\begin{array}{l}42.98 \pm \\
2.44^{\mathrm{a}}\end{array}$ & $\begin{array}{l}42.57 \pm \\
2.59^{\mathrm{a}}\end{array}$ & $\begin{array}{c}43.72 \pm \\
0.91^{\mathrm{a}}\end{array}$ & $\begin{array}{c}38.87 \pm \\
1.87^{\mathrm{a}}\end{array}$ & $\begin{array}{c}36.32 \pm \\
1.37^{\mathrm{a}}\end{array}$ & $\begin{array}{c}41.07 \pm \\
0.87^{\mathrm{a}}\end{array}$ \\
\hline \multicolumn{8}{|l|}{ DLC (\%) } \\
\hline $\begin{array}{l}\text { Neutrophils } \\
(\%)\end{array}$ & $65.76-71.25$ & $\begin{array}{c}66.17 \pm \\
1.54^{\mathrm{a}}\end{array}$ & $\begin{array}{c}67.00 \pm \\
0.68^{*}\end{array}$ & $\begin{array}{c}65.50 \pm \\
1.18^{\mathrm{a}}\end{array}$ & $\begin{array}{c}61.17 \pm \\
4.27^{\mathrm{a}}\end{array}$ & $\begin{array}{l}60.83 \pm \\
2.24^{*}\end{array}$ & $\begin{array}{c}58.17 \pm \\
3.63^{\mathrm{a}}\end{array}$ \\
\hline $\begin{array}{l}\text { Lymphocytes } \\
(\%)\end{array}$ & $5.97-25.75$ & $\begin{array}{c}25.33 \pm \\
1.45^{\mathrm{a}}\end{array}$ & $\begin{array}{c}23.17 \pm \\
0.79^{\mathrm{a}}\end{array}$ & $\begin{array}{c}24.83 \pm \\
0.79^{\mathrm{a}}\end{array}$ & $\begin{array}{c}28.83 \pm \\
4.29^{\mathrm{a}}\end{array}$ & $\begin{array}{c}28.17 \pm \\
2.74^{\mathrm{a}}\end{array}$ & $\begin{array}{l}31.50 \pm \\
3.59^{\mathrm{a}}\end{array}$ \\
\hline $\begin{array}{l}\text { Eosinophils } \\
(\%)\end{array}$ & $2.14-21.31$ & $2.83 \pm 0.3^{\mathrm{a}}$ & $\begin{array}{l}2.50 \pm \\
0.3^{\mathrm{a}}\end{array}$ & $\begin{array}{l}2.67 \pm \\
0.21^{\mathrm{a}}\end{array}$ & $\begin{array}{c}3.00 \pm \\
0.3^{\mathrm{a}}\end{array}$ & $\begin{array}{c}3.00 \pm \\
0.3^{\mathrm{a}}\end{array}$ & $\begin{array}{l}2.50 \pm \\
0.22^{\mathrm{a}}\end{array}$ \\
\hline $\begin{array}{l}\text { Monocytes } \\
(\%)\end{array}$ & $0-5.45$ & $\begin{array}{l}5.33 \pm \\
0.42^{\mathrm{a}}\end{array}$ & $\begin{array}{l}6.67 \pm \\
0.4^{\mathrm{a}}\end{array}$ & $\begin{array}{l}6.33 \pm \\
0.67^{\mathrm{a}}\end{array}$ & $\begin{array}{c}6.00 \pm \\
0.26^{\mathrm{a}}\end{array}$ & $\begin{array}{c}6.83 \pm \\
0.5^{\mathrm{a}}\end{array}$ & $\begin{array}{l}7.17 \pm \\
0.48^{\mathrm{a}}\end{array}$ \\
\hline Basophils (\%) & $0-2.64$ & $\begin{array}{c}0.50 \pm \\
0.22^{\mathrm{a}}\end{array}$ & $\begin{array}{l}0.67 \pm \\
0.21^{\mathrm{a}}\end{array}$ & $\begin{array}{c}0.67 \pm \\
0.21^{\mathrm{a}}\end{array}$ & $\begin{array}{c}0.50 \pm \\
0.22^{\mathrm{a}}\end{array}$ & $\begin{array}{c}0.67 \pm \\
0.21^{\mathrm{a}}\end{array}$ & $\begin{array}{l}0.67 \pm \\
0.21^{\mathrm{a}}\end{array}$ \\
\hline
\end{tabular}

Table.2 Mean \pm SE values of Biochemical parameters of sloth bears in Group A and B

\begin{tabular}{|c|c|c|c|c|c|c|c|}
\hline \multirow[t]{2}{*}{ Parameters } & \multirow{2}{*}{$\begin{array}{c}\text { (Miller and } \\
\text { Fowler, 2003) } \\
\text { 0 min / } \\
\text { Normal range }\end{array}$} & \multicolumn{3}{|c|}{ GROUP A } & \multicolumn{3}{|c|}{ GROUP B } \\
\hline & & 15 minutes & $\begin{array}{c}30 \\
\text { minutes }\end{array}$ & $\begin{array}{c}60 \\
\text { minutes }\end{array}$ & $\begin{array}{c}15 \\
\text { minutes }\end{array}$ & $\begin{array}{c}30 \\
\text { minutes }\end{array}$ & $\begin{array}{c}60 \\
\text { minutes }\end{array}$ \\
\hline ALT (IU / L) & $6-60$ & $\begin{array}{c}14.73 \pm \\
1.50^{\mathrm{a}}\end{array}$ & $\begin{array}{c}13.93 \pm \\
0.77^{\mathrm{a}}\end{array}$ & $\begin{array}{l}12.60 \pm \\
0.82\end{array}$ & $\begin{array}{c}15.70 \pm \\
2.56^{\mathrm{a}}\end{array}$ & $\begin{array}{l}14.00 \pm \\
2.35^{\mathrm{a}}\end{array}$ & $\begin{array}{c}13.17 \pm \\
2.24\end{array}$ \\
\hline AST (IU / L) & $63-234$ & $\begin{array}{l}81.00 \pm \\
5.13^{\mathrm{a}}\end{array}$ & $\begin{array}{c}79.50 \pm \\
6.93^{\mathrm{a}}\end{array}$ & $\begin{array}{c}75.83 \pm \\
5.62\end{array}$ & $\begin{array}{c}74.50 \pm \\
7.11^{\mathrm{a}}\end{array}$ & $\begin{array}{l}72.50 \pm \\
4.43^{\mathrm{a}}\end{array}$ & $\begin{array}{c}70.83 \pm \\
4.62\end{array}$ \\
\hline BUN (mg / dl) & $8-38$ & $\begin{array}{c}14.57 \pm \\
1.65^{\mathrm{a}}\end{array}$ & $\begin{array}{c}14.43 \pm \\
1.84^{\mathrm{a}}\end{array}$ & $\begin{array}{c}14.28 \pm \\
1.77\end{array}$ & $\begin{array}{c}12.08 \pm \\
1.73^{\mathrm{a}}\end{array}$ & $\begin{array}{c}11.60 \pm \\
1.70^{\mathrm{a}}\end{array}$ & $\begin{array}{l}11.51 \pm \\
1.65\end{array}$ \\
\hline $\begin{array}{c}\text { Serum Creatinine } \\
(\mathrm{mg} / \mathrm{dl})\end{array}$ & $0.5-3.0$ & $1.70 \pm 0.21^{\mathrm{a}}$ & $\begin{array}{l}1.70 \pm \\
0.24^{\mathrm{a}}\end{array}$ & $\begin{array}{c}1.62 \pm \\
0.23\end{array}$ & $\begin{array}{l}1.35 \pm \\
0.11^{\mathrm{a}}\end{array}$ & $\begin{array}{l}1.32 \pm \\
0.13^{\mathrm{a}}\end{array}$ & $\begin{array}{c}1.23 \pm \\
0.12\end{array}$ \\
\hline $\begin{array}{c}\text { Total Proteins (g / } \\
\text { dl) }\end{array}$ & $5.4-8.6$ & $6.18 \pm 0.35^{\mathrm{a}}$ & $\begin{array}{l}6.25 \pm \\
0.37^{\mathrm{a}}\end{array}$ & $\begin{array}{c}6.15 \pm \\
0.37\end{array}$ & $\begin{array}{l}6.00 \pm \\
0.39^{\mathrm{a}}\end{array}$ & $\begin{array}{l}5.92 \pm \\
0.32^{\mathrm{a}}\end{array}$ & $\begin{array}{c}5.92 \pm \\
0.29\end{array}$ \\
\hline Glucose (mg/dl) & $48-166$ & $\begin{array}{c}135.9 \pm \\
10.71^{\mathrm{a}}\end{array}$ & $\begin{array}{c}146.6 \pm \\
6.93^{\mathrm{a}}\end{array}$ & $\begin{array}{c}143.0 \pm \\
6.05\end{array}$ & $\begin{array}{l}121.4 \pm \\
9.61^{\mathrm{a}}\end{array}$ & $\begin{array}{l}130.7 \pm \\
10.74^{\mathrm{a}}\end{array}$ & $\begin{array}{c}128.3 \pm \\
10.22\end{array}$ \\
\hline
\end{tabular}

Mean value with superscript $\left(^{a}\right)$ differ non-significantly and superscript $(*)$ differ significantly between columns in Table 1 and 2 
There was no significant difference observed in ALT and AST values indicating the anesthesia having minimum effect on liver cells during biotransformation of drugs similar to reports by Grimm et al., (2011). No significant difference were observed in BUN and Serum Creatinine values although the lesser values in group B could be because of the faster and smoother induction of DexM. It could be also because it is normal for younger animals to have lower BUN and Creatinine values as stated by Graesli et al., (2014). Mean \pm SE of glucose in group A was nonsignificantly higher at 15 and 30 minutes causing urination in group A. This could be because of hyperglycemia caused by Xylazine, as it cause decrease in insulin release from $\beta$ cells and / or increased glucogon from $\alpha$ cells and that Medetomidine cause decrease in Insulin without resultant increase in glucose as reported by Posner and Burn, (2009).

\section{References}

Alfonso, J. and Reis, F., 2012. Dexmedetomidine: Current role in anesthesia and intensive care. Rev. Bras. Anestesiol, 62 (1): 118-133.

Ansah, O. B., 2004. Use of the alpha 2 agonists Medetomidine and Dexmedetomidine in the sedation and analgesia of domestic cats. Academic dissertation. Helsinki. Finland. pp: 1-92.

Arun, A., Jadav, K. K., Illayaraja, S. and Vimal, S., 2008. Hematology of sloth bears (Melursus ursinus ursinus) from two locations in India. J. Wildl. Dis., 44 (2): 509-518

Cattet, M. R. L., Caulkett, N. A. and Lunn, N. J., 2003a. Anesthesia of polar bears using Xylazine-Zolazepam-Tiletamine or Zolazepam-Tiletamine. J. Wildl. Dis., 39 (3): 655-664.

Cattet, M. R. L., Caulkett, N. A. and Stenhouse, G. B., 2003b. Anesthesia of grizzly bears using XylazineZolazepam-Tiletamine or ZolazepamTiletamine. Ursus, 14: 88-93.

Cattet, M. R. L., Christison, K., Caulkett, N. A. and Stenhouse, G. B., 2003c. Physiological responses of grizzly bears to different methods of capture. $J$. Wildl. Dis., 39 (3): 649-654.

Caulkett, N. and Cattet, M. R. L., 2002. Anesthesia of bears. International Veterinary Information Service., www.ivis.org. Retrieved on 7-4-2016.

Fowler, M. E., 1978. Zoo and wild animal medicine. In: Carnivores (carnivore): Ursidae. Wallach, J. WB. Saunders company, USA. Edn. 1., ISBN: 0-72166559-4., pp: 628-637.

Ganong, W. F. "Sistema nervioso autonomo" 2002. In: El Manual Moderno S.A and D.F. Mexico. Fisiologia Medica. Edn. $18^{\text {th }}$. Mexico, $p p:$ 245-252.

Graesli, A. R., Fahlman, A., Evans, A. L., Bertelsen, M. F., Arnemo, J. M. and Nielsen, S. S., 2014. Haematological and biochemical reference intervals for free-ranging brown bears (Ursus arctos) in Sweden. BMC Vet. Res., 10: 183.

Grimm, A. K., Tranquilli, J. W. and Lamont, A. L., 2011. Essentials of small animal anesthesia and analgesia. In: Anaesthesia for patients with liver disease. Wiley Blackwell publication. Edn: 2, pp: 425.

Jin, Y., Qiao, Y., Liu, X., Pu, T., Xu, H. and Lin, D., 2016. Immobilization of wild giant panda (Ailuropoda melanoleuca) with Dexmedetomidine-TiletamineZolazepam. Veterinary Anaesthesia and Analgesia, 43: 333-337. doi:10.1111/vaa.12301.

Miller, R. E and Fowler, M. E., 2003. Zoo and wild animal medicine. In: Ursidae and Hyanidae, Ramsay, E. C. Saunders (Elsevier science). Edn.5. ISBN: 07216-9499-3., pp: 523-538. 
Neto, F. J. T., 2009. Dexmedetomidine: a new alpha-2 agonist for small animal practice. Proceedings of the 34th World Small Animal Veterinary Congress. WSAVA 2009. Sao Paulo, Brazil, pp: 15.

OzekI, L. M., Fahlman, A., Stenhouse, G., Arnemo, J. M. and Caulkett, N., 2014. Evaluation of the accuracy of different methods of monitoring body temperature in anesthetized brown bears (Ursus arctos). J. Zoo Wildl. Med., 45 (4): 819-824.

Posner, L. P. and Burns, P., 2009. Veterinary Pharmacology and Therapeutics. In: Injectable anesthetic agents. In: Sedative agents: Tranquilizers, alpha-2 agonists, and related agents. Wiley
Blackwell publication. Edn. $9^{\text {th }}, p p$ : 265-380.

Rafee, M. A., Kinjavdekar, P., Amarpal, Aithal, H. P., Wani, S. A. and Sangeetha, P., 2015. Global journal of Medical Research (G). 15: issue 1. Version 1.pp: 1-5

Rai, U., 2009. Behavioural study for the conservation breeding of Asiatic black bear (Ursus thibetanus) in Padmaja Naidu Himalayan Zoological Park, Darjeeling. $p p:$ 37-44.

Teisberg, J. E., Farley, S. D., Nelson, O. L., Hilderbrand, G. V., Madel, M. J., Owen, P. A., Erlenbach, J. A. and Robbins, C. T., 2014. Immobilization of grizzly bears (Ursus arctos) with Dexmedetomidine, Tiletamine, and Zolazepam. J. Wildl. Dis., 50 (1): 74-83.

\section{How to cite this article:}

Anitha, M.M., L. Ranganath, H.S. Shwetha, Arun Sha, B.P. Shankar, V. Mahesh and Srinivas, R.B. 2018. Studies on Physiological and Hemato-Biochemical Changes during XylazineZolazepam + Tiletamine and Dexmedetomidine - Zolazepam + Tiletamine Anesthesia in Sloth Bears. Int.J.Curr.Microbiol.App.Sci. 7(12): 3562-3566.

doi: https://doi.org/10.20546/ijcmas.2018.712.403 